Pensamiento Crítico Vol. 21, No 2, pp. 51 - 63

\title{
La inversión extranjera directa en el Perú y sus implicancias en el crecimiento económico 2009-2015
}

Foreign direct investment in Peru and its implications on economic growth 2009-2015

[Recepción: Setiembre de 2016 / Conformidad: Octubre de 2016]

Rafael Bustamante Romaní

\section{RESUMEN}

La economía peruana viene presentando un crecimiento económico sostenido que se ha prolongado ininterrumpidamente por más de dos décadas, pese a mostrar algunos episodios de desaceleración a raíz de las últimas crisis en los mercados financieros internacionales ${ }^{2}$. Dentro de este contexto, un factor que ha contribuido decisivamente al dinamismo de la producción nacional ha sido el flujo de inversión extranjera directa (FIED), que ha significado el ingreso de importantes sumas de capitales del exterior orientadas hacia distintos sectores de nuestra economía, favoreciendo su expansión. Estos flujos de capital se han generado por las

1 Economista y docente de la UNMSM. Magíster en Economía- MBA Centrum PUCP.

2 El 2008 será recordado no solo como el año en que el sistema financiero intemacional implosionó y cayó en una profunda crisis -que aún no sabemos cuánto durará. También se recordará como el año en que los paradigmas político-económicos impuestos desde los años 90 fueron resquebrajados por sus propias y profundas contradicciones. Nos referimos al Consenso de Washington y el neoliberalismo rampante, la obsolescencia del Estado, el auge del capitalismo salvaje, el crecimiento financiero perpetuo, etcétera: la realidad se ha tomado su revancha sobre estas ilusiones, imponiendo un proceso de transformación de las reglas de juego. 


\title{
Pensamiento Crítico Vol. ᄅl, № 2
}

oportunidades que viene ofreciendo el país, como la posibilidad de obtener altas tasas de rendimiento con un entorno económico favorable a la inversión privada y de respeto al libre mercado. En el presente artículo se busca explicar cuál ha sido su impacto en el crecimiento económico a través de un modelo de cointegración entre el producto bruto interno (PBI) y el FIED.

Palabras clave: Crecimiento económico, empleo e inversión, cointegración.

\begin{abstract}
The Peruvian economy has been showing steady economic growth that has continuously extended for more than two decades, despite showing some episodes of slowdown in the wake of the latest crisis in international financial markets. Within this context, a factor that has contributed decisively to the dynamism of domestic production has been the flow of Foreign Direct Investment (FIED), which has meant the entry of large sums of foreign capital oriented towards different sectors of our economy, favoring expansion. These capital flows have been generated by the opportunities that are offering the country, such as the possibility to obtain high rates of return with a favorable economic environment for private investment and respect for the free market. In this article seeks to explain what has been its impact on economic growth it through a model of cointegration between Gross Domestic Product (GDP) and the Flow of Foreign Direct Investment (FIED).
\end{abstract}

Keywords: Economic growth, employment and investment, cointegration.

\section{Introducción}

Durante los ochenta, el FIED permaneció constante; y durante los años noventa, gracias a las reformas estructurales emprendidas en esa época, dicho flujo se ha incrementado en más de 10 veces mientras que los flujos comerciales tan solo se han duplicado. Así, según el World Investment Report, para el año 2000 la inversión extranjera directa se desarrolló con rapidez, aumentando con ello el peso de la producción internacional en la economía mundial. «La IED creció un $18 \%$ en el 2000 , más deprisa que otros agregados económicos tales como la producción, la formación de capital y el comercio mundial y alcanzó la cifra récord de US $\$ 1.3$ miles de millones» (UNCTAD, 2001). 


\section{Rafaєl Bustamante Romaní}

En el 2011 el FIED aumentó en un 16 por ciento con respecto al 2010, lo que refleja las mayores utilidades de las empresas transnacionales y de alto crecimiento económico en los países en desarrollo, cuyo valor se incrementó en un 3 por ciento, alcanzando los $\$ 20.4$ billones (UNCTAD, 2012).

El aumento fue generalizado; abarca a los tres principales grupos de economías desarrolladas, en desarrolloy en transición. Estasúltimas registraron un aumento del 12 por ciento con respecto al año 2010, alcanzando un nivel récord de \$777,000 millones en el 2011, principalmente a través de un aumento sostenido de los nuevos proyectos.

Existen dos posturas para estudiar el papel del FIED; una que se centra en características asociadas al financiamiento de la balanza de pagos, y otra propiamente al desarrollo productivo. La primera adopta generalmente una perspectiva macroeconómica y analiza el fenómeno del FIED en términos del volumen de sus ingresos netos, con un enfoque en el que más es mejor.

En la segunda postura tiene en cuenta al FIED en términos de su contribución a ciertos aspectos microeconómicos, tomando en consideración factores específicos vinculados a los impactos del FIED y de la acción de las empresas transnacionales a la economía local, tales como: i) el surgimiento de nuevas actividades que extienden o profundizan la industrialización; ii) el acceso, la transferencia y la asimilación de tecnologías; iii) el establecimiento y la profundización de encadenamientos productivos; iv) el entrenamiento y capacitación de recursos humanos; v) el desarrollo empresarial local, entre otros (Cepal, 2003).

La literatura sugiere que existe una serie de beneficios que los países receptores de este tipo de inversiones tienen. Mayores flujos de inversión extranjera directa pueden contribuir a una aceleración del crecimiento económico en los países en desarrollo a través de tres mecanismos: (i) aumentos en el stock de capital y, por ende, en la capacidad productiva de la economía, (ii) mayores entradas de divisas que contribuyen a aliviar los desequilibrios externos y, por tanto, a atenuar los efectos nocivos de aquellos sobre el crecimiento, y (iii) transferencias de nuevas técnicas de producción, marketing y administración, que contribuyen a aumentar la productividad general de la economía (Romer, 1993). 


\section{Pensamiento Crítico Vol. ᄅl, № 2}

A nivel agregado, existe evidencia empírica; en este sentido, Borensztein, De Gregorio y Lee (1998) estudian el efecto del FIED de los países industriales sobre 69 países en desarrollo, y encuentran que este afecta positivamente la productividad de las economías receptoras. Además, concluyen que para que estos efectos positivos sean internalizados se requiere que tales economías tengan un stock mínimo de capital humano.

\section{La inversión extranjera directa en el Perú}

La economía peruana viene presentando en los últimos años un crecimiento económico sostenido que se ha prolongado ininterrumpidamente por más de una década, a pesar de presentar algunos episodios de fuerte desaceleración, dentro de un entorno complicado a raíz de las últimas crisis en los mercados financieros internacionales.

Dentro de este contexto positivo, un factor que ha contribuido decisivamente al dinamismo de la producción nacional ha sido el FIED, que ha significado el ingreso de importantes sumas de capitales del exterior orientadas hacia distintos sectores de nuestra economía, incrementando su dinámica de crecimiento. Estos flujos de capital han sido atraídos por las oportunidades financieras y económicas que ha venido ofreciendo el país, tales como la posibilidad de obtener altas tasas de rendimiento, en un entorno favorable a la inversión privada y de libre mercado.

Esas condiciones favorables han originado que el FIED alcance niveles no observados desde hace muchos años. Según datos del Banco Central de Reserva,

nuestro país experimentó el año 2012 un flujo neto de capitales por concepto de FIED ascendente a US\$12,240 millones, cifra que representó poco más del $6 \%$ del PBI. Este dato, además, significó un extraordinario incremento de $49 \%$ de la FIED, con relación al año anterior. Asimismo, el comportamiento del flujo de FIED en Perú pasó de representar alrededor del 1.5\% del PBI en el 2000 a superar el 6\% del PBI el año pasado, lo que da cuenta de la mayor importancia que ha adquirido esta variable en relación con el volumen de la producción doméstica (Scotiabank, 2012). 


\section{Rafaєl Bustamante Romaní}

Los factores que tienen una incidencia positiva sobre la inversión privada incluyen el incremento del FIED y la tendencia decreciente que se dio en el costo del crédito corporativo, que es al que acceden las empresas grandes y medianas.

Asimismo, el Perú registró un FIED récord en el primer semestre del 2012, superando en $31 \%$ al mismo periodo del 2011.

Mucho del FIED nuevo pareciera estar motivado por la percepción de que el Perú se ha vuelto un país, más bien, de riesgo relativamente bajo. Asimismo, la evolución significativa de los flujos de FIED hacia el país ha ocasionado un considerable aumento en el stock de FIED en los últimos años. De acuerdo a cifras del BCRP, proporcionadas por Proinversión, dicho stock asciende a US\$ 63,448 millones a diciembre 2012, monto nominalmente mayor en $24 \%$ a lo reportado al cierre del año anterior y cinco veces superior a lo observado en el 2002.

A continuación, en la figura 1, presentamos el comportamiento de los flujos de inversión extranjera directa durante los periodos 2010 a $2018^{3}$.

\section{FIGURA 1}

Flujo de inversión extranjera directa

(Millones de dólares)

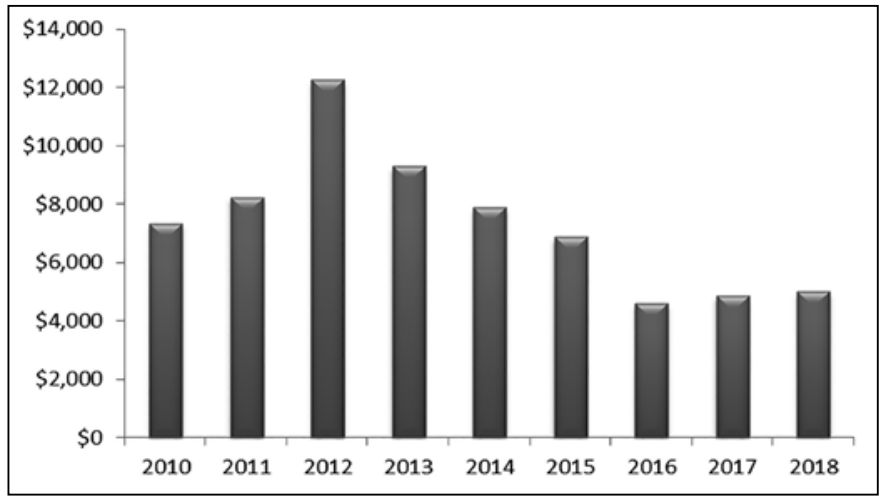

Fuente: ProInversión. Elaboración: ProInversión

3 Años 2016, 2017 y 2018 son proyecciones realizadas por Proinversión. 


\section{Pensamiento Crítico Vol. ᄅl, № 2}

Es preciso destacar también que, con el resultado alcanzado el 2012, la economía peruana se posicionó como la sexta mayor receptora de FIED en América Latina y El Caribe (Cepal, 2012). En dicho documento, la entidad muestra que la región en su conjunto logró un récord histórico de FIED, el cual llegó a US\$174,546 millones, monto que representó un incremento de 5.7\% en comparación con el año 2011 (Cepal, 2012).

\section{Estimación del modelo econométrico}

En esta sección se procede a explicar las etapas para la estimación de un modelo de cointegración aplicando la metodología de cointegración de Johansen.

Los contrastes más frecuentemente utilizados de estacionariedad de la perturbación se realizan a través del análisis de la cointegración entre variables, denominándose variables cointegradas precisamente a aquellas que cumplen determinados requisitos para que la perturbación resultante, al restar a la variable dependiente su valor esperado, sea estacionaria. La perturbación sigue un proceso estacionario, si existe una combinación lineal entre las variables y dada en este caso por la parte determinista del modelo que las relaciona.

\section{Análisis de estacionariedad}

A continuación, se procede a realizar un análisis de estacionariedad de las series para corroborar si son raíces unitarias o integradas de orden uno (véase figura 2).

FIGURA 2

Flujo de inversión extranjera directa

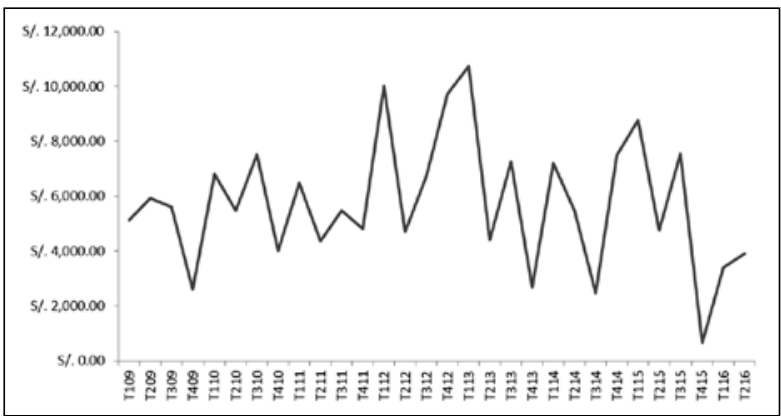

Fuente: BCRP. Elaboración propia. 


\section{Rafaєl Bustamante Romaní}

Se observa que el comportamiento de la serie es típico de una serie estacionaria. Sin embargo, para nuestro análisis necesitamos series integradas de orden uno (raíz unitaria), para ello necesitamos encontrar evidencia de que realmente sea así, dado que la gráfica no es determinante. Para ello hemos recurrido al test de Dickey and Fuller aumentado, estimando el test sin intercepto ni tendencia y obteniéndose los siguientes resultados.

\section{CUADRO 1}

\begin{tabular}{|lcc|}
\hline & t-Statistic & Prob.* $^{*}$ \\
Augmented Dickey-Fuller test statistic & -0.590986 & 0.4497 \\
Test critical values: $5 \%$ level & -1.957204 & \\
*Mackinnon (1996) one-side- p-values. & & \\
\hline
\end{tabular}

Estimaciones realizadas por el autor

El test nos muestra claramente que existe raíz unitaria dado que el valor del estadístico $\mathrm{ADF}$, en valor absoluto, es 0.59 y es menor que el valor de los valores críticos Mackinnon, en valor absoluto, que es de 1.95 .

FIGURA 3

Producto bruto interno, no desestacionalizado

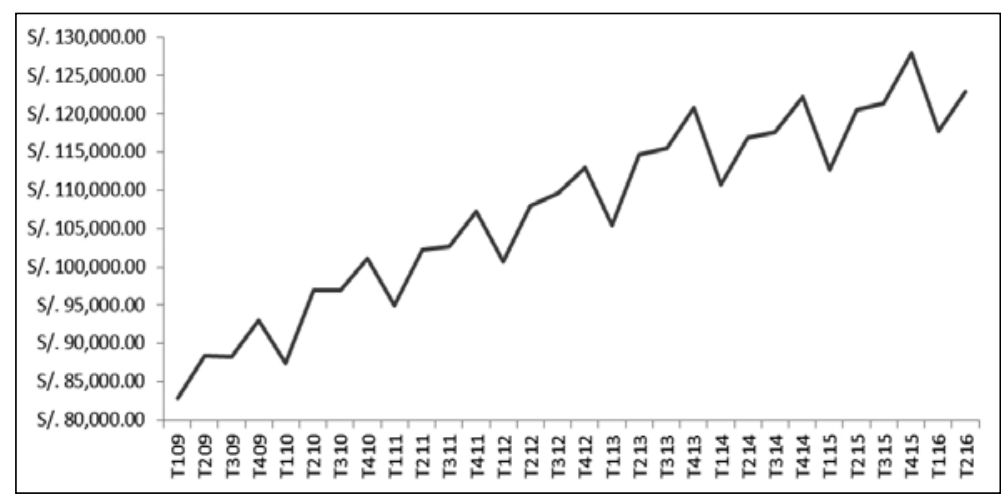

Se observa que el comportamiento de la serie es típico de una serie raíz unitaria con intercepto. Aplicando el test de Dickey and Fuller aumentado, estimado el test con intercepto y sin tendencia, se obtuvieron los siguientes resultados. 


\section{Pensamiento Crítico Vol. ᄅl, № 2}

\section{CUADRO 2}

\begin{tabular}{|lcc|}
\hline & t-Statistic & Prob.* $^{*}$ \\
Augmented Dickey-Fuller test statistic & -1.946951 & 0.3073 \\
Test critical values: 5\% level & -2.967767 & \\
*Mackinnon (1996) one-side- p-values. & & \\
\hline
\end{tabular}

Estimaciones realizadas por el autor

El test nos muestra claramente que existe raíz unitaria dado que el valor del estadístico ADF, en valor absoluto, es 1.94 y es menor que el valor de los valores críticos Mackinnon, en valor absoluto, que es 2.97 .

\section{Pruebas econométricas para determinar el número de rezagos óptimos en el modelo}

Para tener el número de rezagos óptimos del modelo de corrección de errores asociado al modelo de cointegración, procedemos a testear bajo diferentes enfoques.

CUADRO 3

\begin{tabular}{|c|c|c|c|c|c|c|}
\hline \multicolumn{7}{|c|}{$\begin{array}{l}\text { Pruebas de exclusión de rezagados } \\
\text { VAR Lag Order Selection Criteria } \\
\text { Endogenous variables: LNPBI LNIED }\end{array}$} \\
\hline Lag & LogL & LR & FPE & AIC & SC & $\mathrm{HQ}$ \\
\hline 0 & 8.46 & NA & 0.00 & -0.54 & -0.44 & -0.51 \\
\hline 1 & 65.97 & 100.65 & 0.00 & -5.00 & -4.70 & -4.92 \\
\hline 2 & 75 & $14.12 *$ & $1.56 e-05$ & $-5.407 *$ & -4.92 & -5.28 \\
\hline 3 & 75.72 & 1.18 & 0.00 & -5.14 & -4.46 & -4.96 \\
\hline \multicolumn{7}{|c|}{ LR: sequential modified LR test statistic (each test at $5 \%$ level) } \\
\hline \multicolumn{7}{|c|}{ FPF: Final prediction error } \\
\hline \multicolumn{7}{|c|}{ AIC: Akaike information criterion } \\
\hline \multicolumn{7}{|c|}{ SC: Scharwz information criterion } \\
\hline HQ: Hanr & inforn & iterion & & & & \\
\hline
\end{tabular}

Estimaciones realizadas por el autor

Observamos que los distintos test $\mathrm{LR}, \mathrm{FPE}, \mathrm{AIC}, \mathrm{SC}, \mathrm{HQ}$ nos dicen que el número de rezagos óptimo es dos. 


\section{Rafael Bustamante Romaní}

\section{Pruebas de causalidad Granger por bloques}

Para determinar el orden de causalidad, a partir del modelo preliminar de vectores autorregresivos, sometemos a las variables en estudio al test de causalidad Granger por bloques. Aquí la hipótesis nula es que la variable explicativa no causa en el sentido Granger a la variable dependiente.

\section{CUADRO 4}

\begin{tabular}{|c|c|c|c|}
\hline \multicolumn{4}{|c|}{ VAR Granger Causality/Block Exogeneity Waid Tests } \\
\hline Dependent variabl & & & \\
\hline Excluded & Chi-sq & df & Prob. \\
\hline LNIED & 53.4 & 9.0 & 0.0 \\
\hline AII & 53.4 & 9.0 & 0.0 \\
\hline Dependent variabl & & & \\
\hline Excluded & Chi-sq & df & Prob. \\
\hline LNPBI & 14.45 & 9.00 & 0.11 \\
\hline AII & 14.45 & 9.00 & 0.11 \\
\hline
\end{tabular}

Estimaciones realizadas por el autor

\section{Test de cointegración de Johansen}

Según el test de cointegración de Johansen existe evidencia de que al menos existe un vector de cointegración, en sus diferentes versiones y bajo los dos test que plantea el autor (Trace y Max - Eig).

\section{CUADRO 5}

\begin{tabular}{|c|c|c|c|c|c|}
\hline \multicolumn{6}{|c|}{$\begin{array}{l}\text { Series: LNPBILNIED } \\
\text { Lags interval: } 1 \text { to } 2 \\
\text { Selected }(0.05 \text { level*) Number of Cointegrating Relations by Model }\end{array}$} \\
\hline Data Trend: & None & None & Linear & Linear & Quadratic \\
\hline \multirow[t]{2}{*}{ Test Type } & No Intercept & Intercept & Intercept & Intercept & Intercept \\
\hline & No Trend & No Trend & No Trend & Trend & Trend \\
\hline Trace & 1 & 1 & 2 & 1 & 0 \\
\hline Max-Eig & 1 & 1 & 0 & 1 & 1 \\
\hline
\end{tabular}

Estimaciones realizadas por el autor 


\section{Pensamiento Crítico Vol. ᄅl, № 2}

Sin embargo, se decidió optar por elegir el modelo de la cuarta columna; es decir, un modelo con intercepto en el modelo de corrección de errores y tendencia determinística en el vector de cointegración.

\section{Estimación del modelo de cointegración}

A continuación, presentamos el modelo de cointegración estimado bajo la metodología de Johansen, el cual nos muestra un comportamiento inelástico. Es decir, si la inversión crece a una tasa del $1 \%$, en el trimestre, la tasa de crecimiento del producto crecerá en una magnitud del $0.13 \%$.

\section{CUADRO 6}

\begin{tabular}{|lcc|}
\hline Vector de cointegración & \multicolumn{2}{c|}{ t-statistics in [ ] } \\
\hline LNPBI & Variable Dependiente \\
LNIED & \multicolumn{2}{c|}{0.13} \\
& \multicolumn{2}{c|}{$[-4.06]$} \\
@TREND(09Q1) & 0.01 \\
& \multicolumn{2}{c|}{$[-13.9]$} \\
C & \multicolumn{2}{c|}{ 10.69 } \\
\hline Modelo de corrección de errores & D(LNPBI) & 11.4529 \\
\hline CointEq1 & -0.26 & 2.27 \\
& -4.2 & 15.23 \\
D(LNPBI)(-1)) & 0.04 & -1.05 \\
D(LNPBI)(-2)) & 0.13 & 0.02 \\
D(LNIED)(-1)) & -0.01 & 0.08 \\
D(LNIED)(-2)) & 0.00 & -0.19 \\
c & 0.01 & 0.55 \\
R-squared & 0.55 & -4.39 \\
Akaike information criterion & & -3.67 \\
Scharwz criterion & & \\
\hline
\end{tabular}

Estimaciones realizadas por el autor

Asimismo, vemos que los coeficientes asociados al modelo de corrección de errores (-0.26 y 11.45) son significativos. Esto valida el siguiente análisis de las funciones impulso respuesta, tal como se muestra a continuación en la figura 4.

Las funciones impulso respuesta fueron obtenidas a partir del vector de corrección de errores, que utiliza como insumo la ecuación de cointegración entre el producto bruto interno y el flujo de la inversión extranjera directa. 


\section{Rafaєl Bustamante Romaní}

Se puede observar que el FIED tiene un impacto positivo y permanente con tendencia creciente en el mediano plazo. Asimismo, se puede observar que la tasa de crecimiento del PBI tiene un impacto positivo y permanente sobre el FIED, estabilizándose este impacto en $0.20 \%$.

FIGURA 4

Análisis de las funciones impulso respuesta

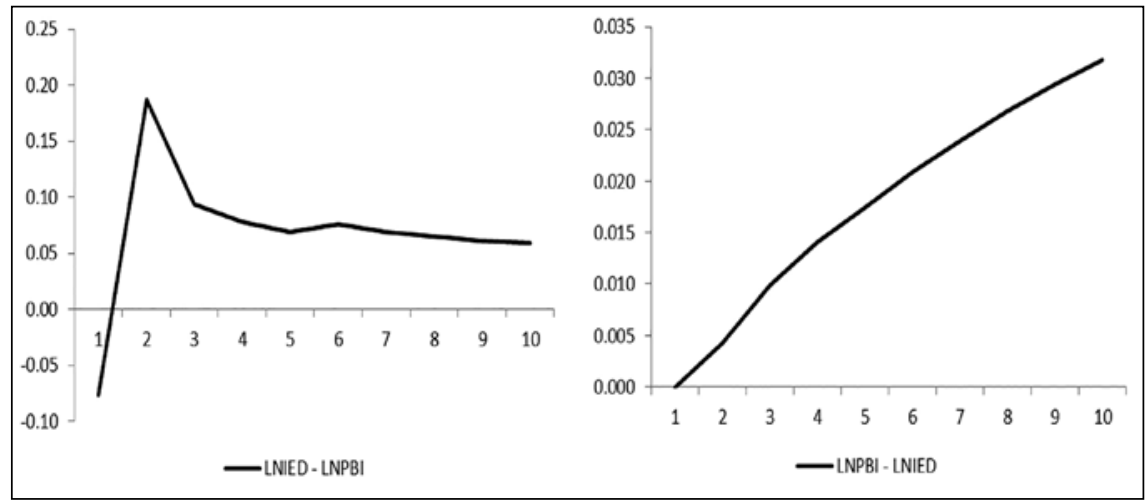

Estimaciones realizadas por el autor

\section{Conclusiones y recomendaciones finales}

1. Después de este análisis descriptivo se puede concluir que es muy cierta la importancia de la inversión extranjera directa como fuente de crecimiento de sectores claves de la economía nacional y mundial. Asimismo, merece destacar el desempeño que ha tenido Perú, sobresaliendo dentro de los países de la región como una economía emergente y atractiva para una afluencia positiva de FIED.

2. Se ha encontrado evidencia empírica de que un incremento en el FIED del $1 \%$ incrementa la tasa de crecimiento del PBI en 1.4\%. Sin embargo, este modelo es preliminar y debe ser mejorado, incorporando otras variables de control como el riesgo país, la tasa de endeudamiento, la evolución de las remuneraciones y la evolución de la productividad de la mano de obra, entre otros; es decir, podríamos tener una idea más clara del impacto del FIED sobre el crecimiento económico del PBI peruano. 


\section{Pensamiento Crítico Vol. ᄅl, № 2}

3. En ese sentido, es fundamental que el país continúe ofreciendo condiciones económicas, políticas y estructurales, adecuadas para atraer los capitales de largo plazo desde el exterior. Esto se logrará no solamente manteniendo reglas de juego claras y sostenibles para el inversionista, sino también defendiendo la institucionalidad y reduciendo las posibles barreras a la inversión privada.

4. Finalmente, como recomendaciones de política, es beneficioso estudiar los principales determinantes del FIED, cuáles son los requisitos institucionales que debe cumplir el país receptor. Entre estos se encuentran el grado de estabilidad política y el grado de intervención del gobierno en la economía, la existencia de derechos de propiedad que determinen los derechos legales que resguarden a las empresas extranjeras de actos arbitrarios de expropiación, ya sea por motivaciones políticas $u$ otras razones. Además de tener muy en cuenta el papel de los efectos generados por esta inversión o externalidades, que tienen mucha importancia como factor explicativo de crecimiento de economías como la asiática.

\section{Referencias bibliográficas}

Álvarez, R. (2002). Inversión extranjera directa en Chile y su impacto sobre la productividad. Santiago: U. Chile Ed.

Asbanc (2013). La inversión extranjera directa, factor del crecimientoeconómicoperuano. ASBANC Semana(73). Disponible en: http://www.asbanc.pe/ContenidoFileServer/ ASBANC\%20SEMANAL\%20N\%C2\%BA73_2013062603123280.pdf

Blomstrom, M.; Nelson, R. y Wolff, E. (1994). Multinational Corporations and Productivity Convergence in Mexico. Convergence of Productivity: Cross-national. (O. a. Press, Ed.) Obtenido de 263-284

Cepal (2003). La inversión extranjera en América Latina y el Caribe. 19-20.

Cepal (2012). La inversión extranjera directa en América Latina y el Caribe. Disponible en: http://www.cepal.org/publicaciones/xml/5/49845/LaInversion ExtranjeraDirectaDoclinf2012.pdf 


\section{Rafael Bustamante Romaní}

Cepal (2012). La Inversión Extranjera Directa en América Latina y el Caribe. Cepal. Disponible en: http://www.eclac.org/publicaciones/xml/5/49845/LaInversion ExtranjeraD2012.pdf

Dagoberto, D. (2013). Notas de Clase del Curso de Finanzas Internacionales. MBA Gerencial CENTRUM Católica.

Haddad, M. (1993). Are There Positive Spillovers from Direct Foreign Investment? 51-74. Disponible en: http://xa.yimg.com/kq/groups/1104317/1896817995/name/ wp06-13bk.pdf

KPMG (2012). Inversiones en el Perú 2012.

Proinversión (2011). Inversión Extranjera Directa. (PROINVERSION, Editor). Disponible en: $\quad$ http://www.proinversion.gob.pe/0/0/modulos/JER/PlantillaStandardsinHijos. aspx?ARE $=0 \& \mathrm{PFL}=0 \& \mathrm{JER}=1537$

Romer, P. (1993). Idea Gaps and Object Gaps in Economic Development. Journal of Monetary Economics, 544-573. Disponible en: http://econ171ucb.files.wordpress. com/2011/06/romer_ideasgap.pdf

Scotiabank (2012). Perú: proyecciones macroeconómicas 2013. Scotiabank, Departamento de Estudios Económicos. Disponible en: http://www.scotiabank.com. pe/i_financiera/pdf/macroeconomico/20121121_mac_es.pdf

UNCTAD (2001). World Investment Report: Promoting Linkages. Ginebra: United. Disponible en: http://unctad.org/en/Docs/wir2001overview_en.pdf

UNCTAD (2012). World Investment Report. New York and Geneva. Disponible en: http://unctad.org/en/PublicationsLibrary/wir2012_embargoed_en.pdf 